\title{
Human single-chain variable fragment antibody inhibits macrophage migration inhibitory factor tautomerase activity
}

\author{
MAYURI TARASUK ${ }^{1}$, ORNNUTHCHAR POUNGPAIR ${ }^{1}$, DUANGPORN UNGSUPRAVATE ${ }^{1}$, \\ KUNAN BANGPHOOMI $^{2}$, WANPEN CHAICUMPA ${ }^{3}$ and PA-THAI YENCHITSOMANUS ${ }^{1}$
}

\author{
${ }^{1}$ Division of Molecular Medicine, Department of Research and Development, Faculty of Medicine Siriraj Hospital, \\ Mahidol University, ${ }^{2}$ Department of Biochemistry, Faculty of Sciences, Kasetsart University, \\ ${ }^{3}$ Laboratory for Research and Technology Development, Department of Parasitology, \\ Faculty of Medicine Siriraj Hospital, Mahidol University, Bangkok, Thailand
}

Received September 13, 2013; Accepted December 24, 2013

\section{DOI: $10.3892 / \mathrm{ijmm} .2014 .1622$}

\begin{abstract}
Macrophage migration inhibitory factor (MIF) is a pro-inflammatory cytokine, secreted from a variety of immune cells, that regulates innate and adaptive immune responses. Elevation of MIF levels in plasma correlates with the severity of inflammatory diseases in humans. Inhibition of MIF or its tautomerase activity ameliorates disease severity by reducing inflammatory responses. In this study, the human single-chain variable fragment $(\mathrm{HuScFv})$ antibody specific to MIF was selected from the human antibody phage display library by using purified recombinant full-length human MIF (rMIF) as the target antigen. Monoclonal $\mathrm{HuScFv}$ was produced from phage-transformed bacteria and tested for their binding activities to rMIF by indirect enzyme-linked immunosorbent assay as well as to native MIF by western blot analysis and immunofluorescence assay. The $\mathrm{HuScFv}$ with highest binding signal to rMIF also inhibited the tautomerase activities of both rMIF and native MIF in human monoblastic leukemia (U937) cells in a dose-dependent manner. Mimotope searching and molecular docking concordantly demonstrated that the $\mathrm{HuScFv}$ interacted with Lys32 and Ile64 in the MIF tautomerase active site. To the best of our knowledge, this is the first study to focus on MIF-specific fully-human antibody fragment with a tautomerase-inhibitory effect that has potential to be developed as anti-inflammatory biomolecules for human use.
\end{abstract}

\section{Introduction}

Macrophage migration inhibitory factor (MIF) was identified in 1966 as the first T-cell cytokine inhibiting random movement

Correspondence to: Professor Pa-Thai Yenchitsomanus, Division of Molecular Medicine, Department of Research and Development, Faculty of Medicine Siriraj Hospital, Mahidol University, 4th Floor SiMR Building, 2 Prannok Rd, Bangkoknoi, Bangkok 10700, Thailand E-mail: ptyench@gmail.com

Key words: macrophage migration inhibitory factor, tautomerase activity, human single-chain variable fragment antibody, inflammation, inflammatory disease of macrophages (1). The biological and physiological activities of human MIF were elucidated from studies on recombinant human MIF generated by molecular techniques. Structural analysis demonstrated that MIF is a homotrimeric molecule with similar characteristics and activities to the human $D$-dopachrome tautomerase (D-DT) enzyme (2). MIF is considered a multifunctional protein constitutively expressed and stored in preformed cytoplasmic pools in several immune cells including monocytes, macrophages, $\mathrm{T}$ and $\mathrm{B}$ lymphocytes, eosinophils, neutrophils, and dendritic cells and is rapidly released in response to stimuli. In addition, MIF has been identified in various tissues and organs such as lung, the epithelial lining of the skin, gastrointestinal and genitourinary tracts (reviewed in ref. 3).

MIF plays a pivotal role in immune inflammatory responses by acting as a major mediator in the counter-regulation of the immunosuppressive effects of glucocorticoids. As it is an upstream regulator, MIF exerts its effects in innate and adaptive immune systems by the activation of leukocyte recruitment and initiation of the inflammatory cascade for the release of other pro-inflammatory cytokines and mediators such as interleukin (IL)-1 $\beta$, IL-2, IL-6, IL- 8, TNF- $\alpha$, nitric oxide and prostaglandin E2 (3-5). Furthermore, MIF inhibits p53-mediated apoptosis of immune cells during inflammatory activation (6). MIF biological activity correlates with several catalytic activities such as D-DT (7), phenylpyruvate tautomerase (8), and thiol-protein oxidoreductase (9). However, its physiological substrate has yet to be identified.

Clinical studies have identified the correlation between MIF levels in plasma or affected tissue and disease severity in a number of inflammation-associated diseases. Elevated levels of MIF have been detected in sepsis (10) as well as numerous infectious diseases with exaggerated immune response such as dengue hemorrhagic fever $(11,12)$ and influenza virus infection (13). An increase of secreted MIF has also been observed in a number of inflammatory-mediated autoimmune and also metabolic diseases (14-19). In addition, MIF has a direct relationship with cancer growth and progression (20). Therefore, MIF has become a promising biomarker for diagnosis and an attractive therapeutic target for the treatment of a wide variety of diseases. 
Inhibition of MIF activities by small molecules or neutralizing antibodies has become crucial in identifying therapeutic strategies to alleviate immuno-inflammatory disorders in humans. It has been well documented that interference of MIF tautomerase activity readily reduces inflammatory-mediated pathogenesis in various disease models such as sepsis, diabetes and allergic neuritis (21-24). Similar to MIF inhibitors, neutralizing anti-MIF antibodies have been proven to be therapeutically effective in several models of autoimmune and inflammatory diseases (25-29). Recently, antibodies specific to $\beta$-sheet structure containing the oxidoreductase motif of MIF have been demonstrated for their protective effects in sepsis model or contact hypersensitivity (30). In the present study, fully human single-chain variable fragment $(\mathrm{HuScFv})$ antibody, which is five-fold smaller than the normal antibody molecule, specific to MIF has been generated. HuScFv selected from a human antibody phage display library, not only recognizes human MIF, but also neutralizes its tautomerase activity. The results of several biological experiments indicate the potential development of MIF-specific $\mathrm{HuScFv}$ for therapeutic or diagnostic applications.

\section{Materials and methods}

Cell culture and native protein preparation. Human monoblastic leukemia (U937) cells were cultured in RPMI-1640 medium (Gibco, Grand Island, NY, USA) supplemented with $10 \% \mathrm{FBS}$ ( $\mathrm{vol} / \mathrm{vol}$ ), $2 \mathrm{mM} \mathrm{L}$-glutamine and $100 \mathrm{U} / \mathrm{ml}$ antibiotics at $37^{\circ} \mathrm{C}$ in a $5 \% \mathrm{CO}_{2}$ atmosphere. Native MIF in U937 cell lysate was used as an antigen in the western blot analysis and experiments regarding tautomerase activity.

Production and purification of recombinant human MIF (rMIF). Full-length human MIF was amplified from kidney Matchmaker cDNA library (Clontech, Mountain View, CA, USA) by using $M I F$-specific primers (forward, 5'-CGG GAT CCA TGC CGA TGT TCA TCG TAA ACA CC-3' and reverse, 5'-CCG CTC GAG GGC GAA GGT GGA GTT GTT C-3'). BamHI and XhoI endonuclease restriction sites (underlined) were incorporated for DNA cloning purposes. The amplicon was digested and ligated into pET21a(+) vector (Novagen, Darmstadt, Germany) and introduced into $E$. coli BL21(DE3). A colony of transformant E. coli carrying MIF was induced by IPTG for rMIF production. Polyhistidine-tagged-rMIF was purified by TALON ${ }^{\mathrm{TM}}$ Metal Affinity Resin (Clontech) under native conditions. The purity of rMIF was determined by $15 \%$ SDS-PAGE and Coomassie Brilliant Blue G-250 (Sigma, St. Louis, MO, USA) staining.

Phage bio-panning. Phage clones carrying MIF-specific $\mathrm{HuScFv}$ were selected from the human antibody phage display library by bio-panning procedure (31). Purified $\operatorname{rMIF}(1 \mu \mathrm{g})$ was coated into microtiter wells and phage library $(100 \mu \mathrm{l}$ containing $\sim 10^{11} \mathrm{pfu}$ ) was added. Phages exhibiting $\mathrm{HuScFv}$ that bound to rMIF were rescued by E. coli HB2151 infection and selected on selective agar plates (LB containing $100 \mu \mathrm{g} / \mathrm{ml}$ ampicillin and $2 \%$ glucose). Individual phagemid-transformed $E$. coli clones were screened for the presence of $h u s c f v$ in a phagemid vector by colony PCR using phagemid-specific primers induced for monoclonal $\mathrm{HuScFv}$ production, as previously described (31). Bacterial lysates were detected for E-tagged $\mathrm{HuScFv}$ by western blot analysis using anti-E-tag polyclonal antibody
(Abcam, Cambridge, UK) followed by HRP-conjugated swine anti-rabbit Ig (Dako, Glostrup, Denmark) and DAB substrate.

Screening of MIF-specific HuScFv by indirect enzyme-linked immunosorbent assay (ELISA). Indirect ELISA was performed to determine the binding of monoclonal $\mathrm{HuScFv}$ to rMIF. The wells of ELISA plate were coated with $1 \mu \mathrm{g}$ purified rMIF or $\mathrm{BSA}$ (negative antigen control) at $37^{\circ} \mathrm{C}$ overnight. After washing and blocking the wells, $\mathrm{HuScFv}$-containing preparations $(1 \mathrm{mg}$ in $100 \mu \mathrm{l}$ ) were added individually to both rMIF and BSA wells and incubated at $37^{\circ} \mathrm{C}$ for $2 \mathrm{~h}$. $\mathrm{HuScFv}$ binding to rMIF was detected by rabbit anti-E-tag polyclonal antibody followed by HRP-conjugated swine anti-rabbit IgG. Enzymatic reaction was developed following the addition of TMB substrate (Invitrogen, Camarillo, CA, USA) and $1 \mathrm{~N} \mathrm{HCl}$. Color of the content in the wells was measured at $\mathrm{OD}_{450 \mathrm{~nm}}$ using ELISA reader (Multiskan EX; Thermo Scientific, Waltham, MA, USA).

Preparation of purified 6xHis-tagged HuScFv. The Huscfv sequence in the phagemid vector of the selected $E$. coli clone was subcloned into modified pET23b(+) vector and introduced into E. coli $\mathrm{BL} 21(\mathrm{DE} 3)$ by transformation (32). Bacterial transformants containing pET23b(+)-huscfv were induced with IPTG for the production of monoclonal 6xHis-tagged HuScFv. The HuScFv in the bacterial lysate was purified using TALON Metal Affinity Resin and prepared in 1X PBS (pH 7.4) by dropwise dialysis prior to use.

Determination of the binding activity of $H u S c F v$ to native $M I F$. Western blot analysis and immunofluorescence assay were performed to determine the binding activity of $\mathrm{HuScFv}$ to native MIF in human U937 cells. U937 whole cell lysate $(40 \mu \mathrm{g})$ was separated on SDS-PAGE and transferred onto nitrocellulose membrane. Polyhistidine-tagged $\mathrm{HuScFv}$ was added to the membrane and subsequently detected by mouse anti-His antibody. The reactive band of $\mathrm{HuScFv}-\mathrm{MIF}$ immune complexes was revealed by adding AP-conjugated goat antimouse Ig and BCIP/NBT colorimetric substrate, respectively. The irrelevant $\mathrm{HuScFv}$ (dengue virus capsid protein-specific $\mathrm{HuScFv}$ ) and mouse anti-MIF polyclonal antibody were used as negative and positive antibody controls, respectively.

Immunofluorescence assay was used to demonstrate and localize the interaction of HuScFv to cellular MIF in U937 cells. The cells were fixed with $4 \%$ paraformaldehyde and permeabilized with $0.2 \%$ Triton X-100. After blocking, the cells were incubated with purified $\mathrm{HuScFv}(1 \mu \mathrm{M})$ at $37^{\circ} \mathrm{C}$ for $2 \mathrm{~h}$ in a humidified chamber. The HuScFv-MIF interaction was revealed by adding a mixture of mouse anti-His antibody and rabbit anti-MIF polyclonal antibody. The cells were then incubated with a mixture of Alexa Flour 488-conjugated goat anti-mouse Ig (Molecular Probes, Carlsbad, CA, USA), Cy ${ }^{\mathrm{TM}} 3$-conjugated AffiniPure donkey anti-rabbit Ig (Jackson ImmunoResearch Laboratories, West Grove, PA, USA), and anti-nuclear staining reagent (Hoechst; Molecular Probes) to localize HuScFv, endogenous MIF, and nuclear DNA, respectively. Fluorescence images were visualized by using a laser scanning confocal microscope (LSM 510 META; Carl Zeiss, Jena, Germany).

Neutralization of MIF tautomerase activity by $\mathrm{HuScFv}$. Recombinant MIF and native MIF in U937 cell lysates were 
analyzed for its inherent tautomerase activity as previously described with slight modifications (33). The enzymatic reaction was initiated at $25^{\circ} \mathrm{C}$ by adding $20 \mu 1$ of the dopachrome methyl ester substrate ( $2 \mathrm{mM}$ L-3,4-dihydroxyphenylalanine methyl ester and $4 \mathrm{mM}$ sodium periodate) to a cuvette containing $200 \mu \mathrm{l}$ of either $\operatorname{rMIF}(30 \mu \mathrm{M})$ or native MIF in U937 cell lysate $(6 \mu \mathrm{g})$ prepared in tautomerase assay buffer $(50 \mathrm{mM}$ potassium phosphate, $1 \mathrm{mM}$ EDTA, pH 6.0). The activity was determined by the semi-continuous reduction of $\mathrm{OD}_{475 \mathrm{~nm}}$ at $60-\mathrm{sec}$ interval for $300 \mathrm{sec}$ using a spectrophotometer (Shimadzu, Kyoto, Japan). The neutralization of tautomerase activity by MIF-specific $\mathrm{HuScFv}$ was determined by pre-incubation of $\mathrm{HuScFv}$ with rMIF or native MIF in U937 cell lysate at $37^{\circ} \mathrm{C}$ for $1 \mathrm{~h}$ prior to the enzymatic reaction was initiated. Irrelevant $\mathrm{HuScFv}$ was used as a negative antibody control. Heat-denatured rMIF and U937 cell lysate were used as tautomerase activity-negative controls. Results were presented as the mean values from three independent experiments.

HuScFv mimotope searching and in silico analysis of $\mathrm{HuScF}$-MIF interaction. Mimotope of MIF-specific HuScFv was determined by using Ph.D.-12 ${ }^{\mathrm{TM}}$ Phage Display Peptide Library (New England Biolabs; Ipswich, MA, USA) as previously described (34). Purified $\mathrm{HuScFv}$ was used as the target for selection of phage clones displaying 12-mer peptide (mimotope). After three rounds of bio-panning, genomic DNA from a number of phage clones displaying peptides bound to MIF-specific HuScFv was sequenced and the 12-mer peptide sequences were deduced. Consensus mimotope was obtained by multiple alignments of the deduced peptide sequences. The mimotope was aligned to the MIF protein sequence and residues on the MIF molecule matching with the obtained mimotope were considered as the $\mathrm{HuScFv}$ epitope.

Molecular modeling of the antibody was initiated via BLAST search analysis. The highest identity sequence with 3D structure was used as a template for homology modeling by using the modeler module embedded in the Discovery Studio 2.5 program (Accelrys Software Inc., San Diego, CA, USA). The three-dimensional structure of MIF was obtained from the PDB database (PDB entry 1GD0) and the crystal structure of a human anti-SARS spike protein antibody, 80R (PDB entry 2GHW) was used as a template for modeling of the $\mathrm{HuScFv}$ molecule. The constructed models were accessed for the quality of structure via the Ramachandran plot. The plot was generated using PROCHECK v3.4 program (University College London, London, UK) (35). Subsequently, the docked poses were subjected to structural refinements by using RDOCK module embedded in Discovery Studio 2.5 program.

Statistical analysis. Data were analyzed using GraphPad Prism Software 4.0 (GraphPad Software, Inc., San Diego, CA, USA) and presented as the mean \pm SEM. Significant differences were determined by one-way ANOVA $(\mathrm{P}<0.05)$ and Tukey's HSD test.

\section{Results}

Production of rMIF with inherent enzymatic activity. A full-length cDNA encoding MIF was amplified from human cDNA library (345 bp). Following DNA sequencing, the sequence was deposited into the GenBank database (accession no. JQ846015). It showed $100 \%$ homology to the human MIF-coding sequence previously reported in the database (NM_002415.1). Recombinant MIF was abundantly expressed after induction of the bacterial clone carrying recombinant plasmid. Polyhistidine-tagged rMIF was purified as a soluble protein at $12.5 \mathrm{kDa}$. Tautomerase enzymatic activity, a distinct pathogenesis-related property of MIF, was verified as the reduction of dopachrome methyl ester substrate at $\mathrm{OD}_{475 \mathrm{~nm}}$ (33). Purified rMIF exhibited in vitro tautomerase activity, suggesting that rMIF presented inherent physiological function, and retained enzymatic epitope similar to the native molecule (data not shown).

Selection and screening of MIF-specific HuScFv. Purified rMIF was used as the antigen for selection of phages carrying MIF-specific HuScFv by bio-panning of the human antibody phage display library. A number of $E$. coli clones carrying $h u s c f v$ were obtained and randomly tested for the production of monoclonal soluble HuScFv. Among huscfv-positive transformants, 28 clones (35\%) produced HuScFv (20-35 kDa) as detected by western blot analysis using anti-E-tag antibody (Fig. 1A). These clones were individually screened for their binding activities to rMIF by indirect ELISA. Seventeen clones exhibiting ELISA signal ratios $\left(\mathrm{OD}_{450 \mathrm{~nm}}\right.$ of $\mathrm{HuScFv}$-rMIF binding/OD ${ }_{450 \mathrm{~nm}}$ of HuScFv-BSA binding) were higher than the ELISA signal ratio identified in the negative control (Fig. 1B). Huscfv of E. coli clones exhibiting high binding activity were subcloned into a modified $\mathrm{pET} 23 \mathrm{~b}(+)$ vector for benefits in $\mathrm{HuScFv}$ purification and detection in subsequent experiments. Only the HuScFv of clone no. $22(\sim 25 \mathrm{kDa})$ was produced in an sufficient amount and appropriate purity for subsequent experiments as revealed by SDS-PAGE and western blot analysis detected with anti-6xHis antibody (Fig. 2A).

Characterization of MIF-specific HuScFv. The HuScFv no. 22 was demonstrated for its binding activity to native MIF by western blot analysis and immunofluorescence staining. Western blot analysis revealed a reactive band of native MIF from U937 cell lysate bound to $\mathrm{HuScFv}$ at the same size as that of the native human MIF protein (12.5 kDa), similar to the positive antibody control (mouse anti-MIF pAb), while the irrelevant $\mathrm{HuScFv}$ showed no reactive band (Fig. 2B). Immunofluorescence staining confirmed the binding activity of HuScFv to intracellular MIF in U937 cells. Co-localization of MIF and HuScFv throughout the cytoplasm of U937 cells was clearly observed under confocal microscopy (Fig. 2C).

Inhibition of MIF tautomerase activity by $\mathrm{HuScF} v$. Inhibitory effect of $\mathrm{HuScFv}$ on MIF dopachrome tautomerase activity was determined. Following pre-incubation with $\mathrm{HuScFv}$, tautomerization reaction indicated by substrate decolorization of both rMIF and native MIF was reduced compared to that of the non-HuScFv control reaction. Increased inhibition was evident with the increasing amount of $\mathrm{HuScFv}$ present in the reaction. At the end-point of rMIF-mediated enzymatic reaction, $\mathrm{HuScFv}$ levels at concentrations of 6,8 and $10 \mu \mathrm{M}$ markedly reduced tautomerase activity to 86,46 and $10 \%$, respectively, compared to the non-HuScFv control reaction (Fig. 3A). On 


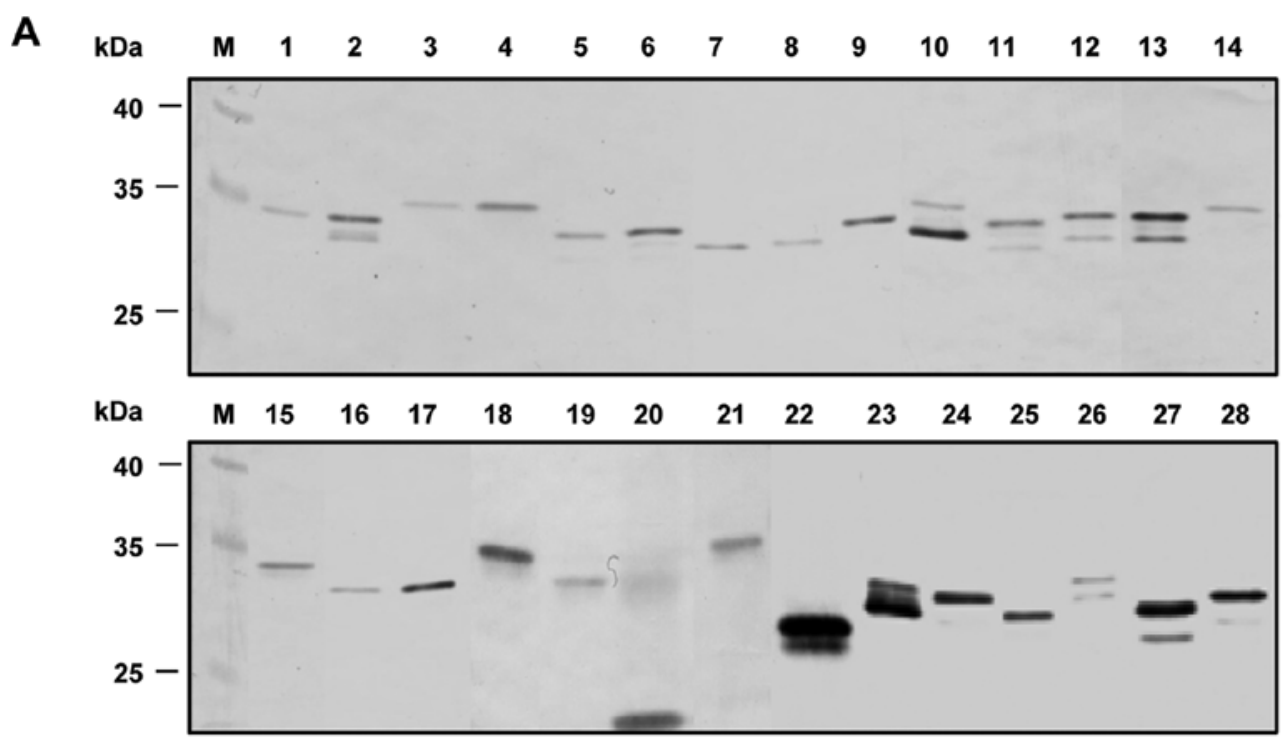

B

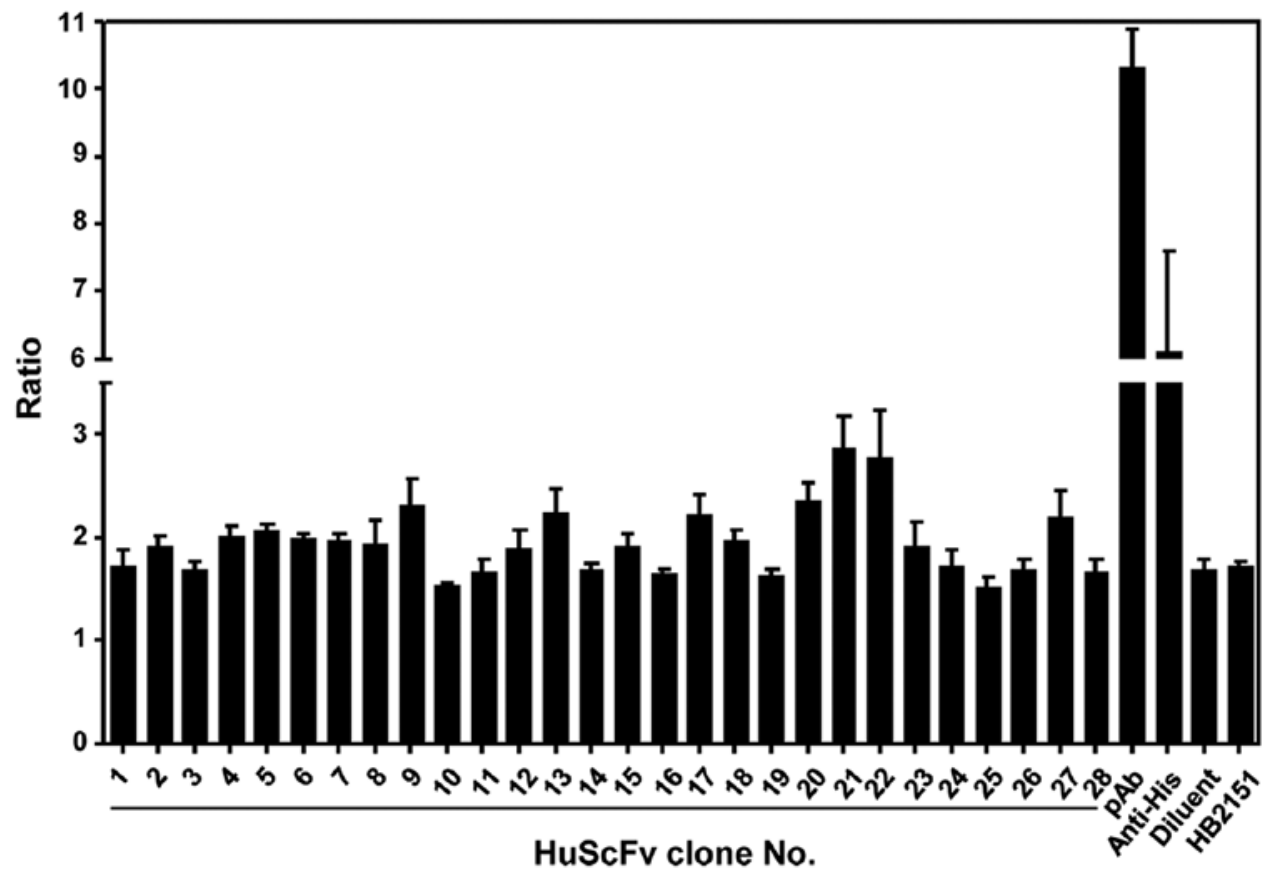

Figure 1. Screening of phage clones displaying migration inhibitory factor (MIF)-specific human single-chain variable fragment (HuScFv) and determination of HuScFv binding activity to recombinant human MIF (rMIF). (A) After bio-panning, monoclonal soluble HuScFv produced from huscfv phagemid-transformed HB2151 E. coli was detected by western blot analysis using the anti-E-tag antibody. The result revealed HuScFv (20-35 kDa) production from 28 E. coli clones. (B) Indirect enzyme-linked immunosorbent assay (ELISA) was used to determine the binding activities of $28 \mathrm{HuScFv}$ clones to rMIF. The results were presented as an average ratio of the binding signal of HuScFv-rMIF compared to HuScFv-BSA $\left(\mathrm{OD}_{450 \mathrm{~nm}}\right.$ of HuScFv-rMIF/OD $450 \mathrm{~nm}$ of $\left.\mathrm{HuScFv}-\mathrm{BSA}\right) \pm \mathrm{SEM}$. Mouse anti-MIF polyclonal antibody and anti-His antibody were used as positive antibody controls. Non-HuScFv-producing $E$. coli lysate served as the negative antibody control.

the other hand, heat-denatured and irrelevant HuScFv did not significantly affect the enzymatic activity of rMIF.

The HuScFv selected from rMIF was also tested for its inhibition of tautomerase activity mediated by native MIF in human monoblastic leukemia (U937) cells. Tautomerase activity of MIF in U937 cells was reduced as MIF-specific $\mathrm{HuScFv}$ was added prior to initiation of enzymatic reaction. As the HuScFv concentration increased $(1.5,5$ and $15 \mu \mathrm{M})$ the average percentages of tautomerase activity were reduced to 99,68 and $44 \%$, respectively (Fig. 3B). By contrast, denatured MIF-specific and irrelevant HuScFv failed to hinder tautomerase activity.
Mimotope searching and in silico analysis of $\mathrm{HuScFv}-\mathrm{MIF}$ interaction. Human MIF-HuScFv interaction was analyzed by means of mimotope searching and molecular docking. In mimotope searching, 20 phage clones carrying peptide mimotopes of $\mathrm{HuScFv}$ were obtained and the 12-mer consensus mimotope sequence was 'MSTPLGQYTGTK'. HuScFv mimotope was aligned on the MIF sequence and it was found to locate at the residues 22-33 of the human MIF-residing tautomerase active site.

In molecular docking, $\mathrm{HuScFv}$ was modeled using a human anti-SARS spike protein antibody with $75 \%$ sequence similarity to the template. Ramachandran plot of the modeled 
A

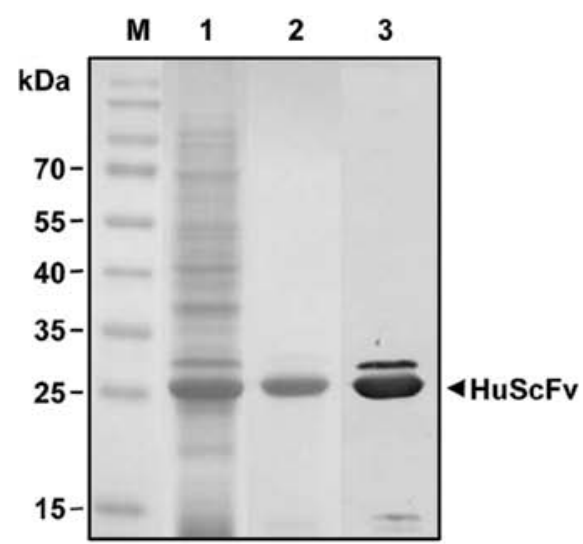

B

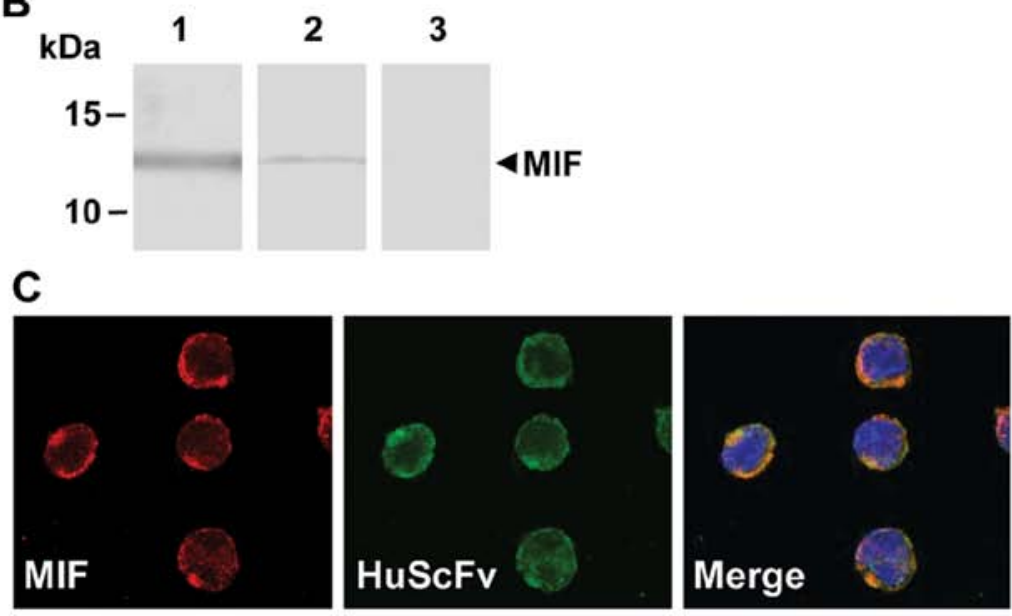

Figure 2. Production of purified migration inhibitory factor (MIF)-specific human single-chain variable fragment (HuScFv) and its binding activity against native MIF. (A) SDS-PAGE and Coomassie Brilliant Blue G-250 staining patterns of E. coli lysate containing 6xHis-tagged HuScFv of clone no. 22 (lane 1) and the affinity-purified $\mathrm{HuScFv}$ (lane 2) were revealed. Western blot analysis indicated HuScFv of clone no. 22 (25 kDa) as detected with anti-His antibody (lane 3). (B) Western blot analysis for determining the binding activity of $\mathrm{HuScFv}$ to native MIF. U937 lysate (40 $\mu \mathrm{g}$ ) was blotted onto nitrocellulose membrane and detected with either i) mouse anti-MIF polyclonal antibody (lane 1), ii) MIF-specific HuScFv followed by mouse anti-His antibody (lane 2), or iii) dengue capsid-specific $\mathrm{HuScFv}$ followed by mouse anti-His antibody (lane 3). Reactive bands of native MIF were observed at size $12.5 \mathrm{kDa}$. (C) HuScFv co-localized with native MIF in U937 cells. Cells were fixed and immunostained with MIF-specific HuScFv as observed in green fluorescence. Rabbit anti-MIF polyclonal antibody indicated intracellular MIF (red fluorescence). The combined image demonstrated co-localization of MIF-specific HuScFv and native MIF as yellow fluorescence throughout the cytoplasm of U937 cells.

A

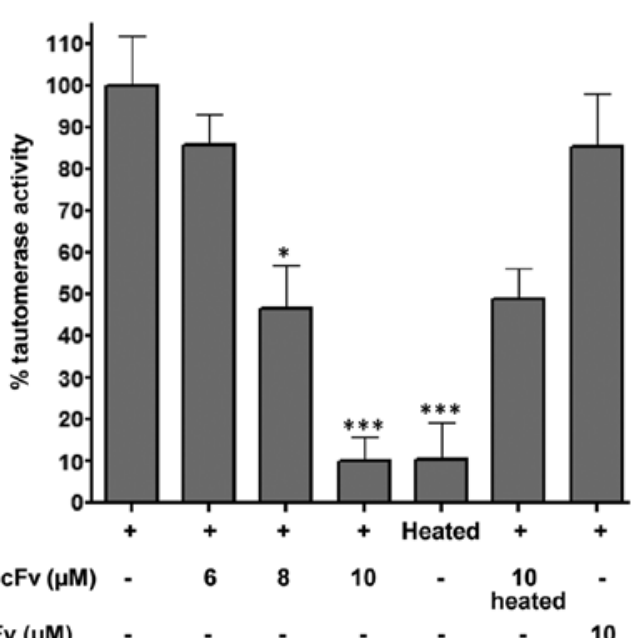

B

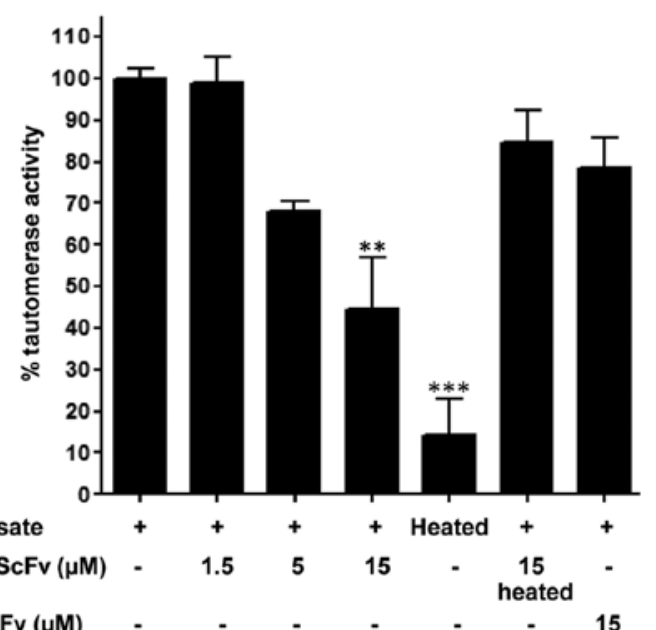

Figure 3. Neutralization of migration inhibitory factor (MIF) tautomerase activity by human single-chain variable fragment (HuScFv). Relative tautomerase activity of (A) recombinant human MIF (rMIF) and (B) native MIF from U937 cells in the presence of HuScFv at various concentrations were shown. Heatdenatured MIF, heat-denatured HuScFv, and irrelevant HuScFv (C-HuScFv; dengue virus capsid-specific HuScFv) were used as negative controls. Data are presented as mean \pm SEM of three independent experiments $\left({ }^{*} \mathrm{P}<0.05,{ }^{* * *} \mathrm{P}<0.01\right.$ and ${ }^{* * * *} \mathrm{P}<0.001$ vs. control; rMIF or U937 cell lysate alone).

$\mathrm{HuScFv}$ revealed the acceptable disallowed region $(1.0 \%)$ indicating that the modeled structure is appropriate for the molecular docking analysis. The docked pose calculated by ZDOCK and RDOCK shown in Fig. 4, exhibited low-binding energy of HuScFv-MIF binding $(-17.493 \mathrm{kcal} / \mathrm{mol})$ at residues Lys32 and Ile64 of the MIF tautomerase active site.

\section{Discussion}

The correlation of increased MIF levels in plasma with severity of inflammatory diseases has been previously reported. Thus, MIF is considered a promising biomarker (36) and therapeutic target for these diseases. It has been shown that interference or abrogation of enzymatic activity of MIF rescued individuals that succumbed to inflammation-mediated pathogenesis $(21,37)$. A number of inhibitors and neutralizing antibodies have been introduced for the inhibition of MIF activity. Recently, Kerschbaumer et al (30) generated fully human anti-MIF antibodies by selection from a phage display library and extensively analyzed these antibody molecules in vitro and in vivo. Results of that study showed that only the antibody-binding epitopes within amino acids 50-68 or 86-102 of the MIF molecule, the regions forming a $\beta$-sheet structure in the MIF oxidoreductase motif, exerted protective effects in models of sepsis or contact hypersensitivity. Findings of this study have demonstrated that fully human antibody fragments specific to MIF inhibited the 


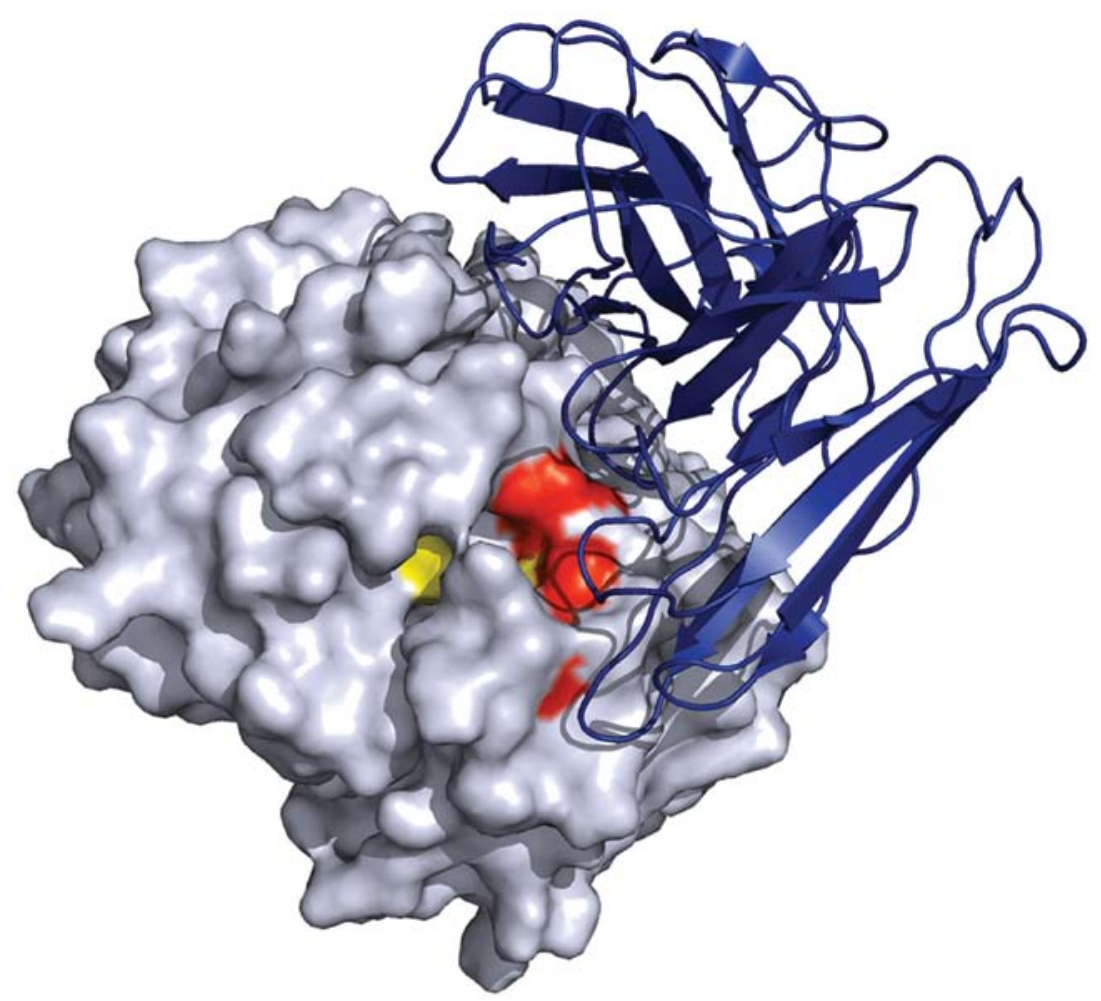

Figure 4. 3D structure of migration inhibitory factor (MIF)-human single-chain variable fragment (HuScFv) complexes. Complex structure of HuScFv (blue ribbon) and trimeric MIF (gray surface) is shown. The tautomerase active site residues are shown in yellow and red surfaces. Mimotope searching and molecular docking results indicated that HuScFv interacted with Lys32 and Ile64 within the tautomerase active site (yellow surface) in the MIF molecule.

enzymatic activity of MIF and presented therapeutic potentials. Identification of HuScFv specific to MIF is also crucial. Unlike the previous study (30), we identified and reported HuScFv that inhibits tautomerase activity of MIF.

In this study, we initially amplified the coding sequence of human MIF from the human cDNA library, which showed $100 \%$ homology to the human MIF-coding sequence previously reported in the NCBI database (NM_002415.1). The rMIF generated from the human MIF cDNA construct exhibited tautomerase activity, suggesting that it carried a native structure and active enzymatic sites; thus, it was suitable for additional use as the target antigen for selection of MIF-specific $\mathrm{HuScFv}$ from the human antibody phage display library. This library, constructed in our laboratory, has previously been used for the selection of a number of neutralizing $\mathrm{HuScFv}$ specific to different pathogenic antigens $(31,32,38,39)$. However, it has not been previously used for the selection of $\mathrm{HuScFv}$ specific to a human protein.

Naturally, immature B lymphocytes carrying antibodies specific to human autologous proteins are eliminated during B-lymphocyte maturation; consequently, they are absent from the pool of circulating lymphocytes. However, results of this study have demonstrated that the human antibody phage display library prepared from human B lymphocytes is composed of $\mathrm{HuScFv}$ specific to human MIF that should be deleted from the matured B lymphocytes. This finding is attributable to the fact that in the generation step of human antibody phage display library the heavy and light chains $(V H$ and $V L)$ of antibody genes were individually amplified and allowed to randomly fuse to form $\mathrm{HuScFv}$. Thus, the combination of $\mathrm{VH}$ and VL that did not exist in nature was available in the in vitro preparation of synthetic antibody phage display library. This explains the fact that a smaller number of huscfv-positive phagemid-transformed E. coli from MIF-bio-panning (29\%) was obtained when they were compared to those from the selection of $\mathrm{HuScFv}$ specific to pathogenic proteins reported in previous studies $(59-100 \%)(31,34,38)$.

Some of the HuScFv clones obtained exhibited strong binding activity to rMIF as determined by indirect ELISA. The $\mathrm{HuScFv}$ with high binding signals were produced and purified. Only $\mathrm{HuScFv}$ of clone no. 22 yielded sufficient amount with appropriate purity, and it was subsequently characterized by results of different experiments. Western blot analysis revealed that $\mathrm{HuScFv}$ no. 22 bound to native MIF protein prepared from the monocytic cell line, U937. Moreover, immunofluorescence staining revealed co-localization of $\mathrm{HuScFv}$ and native MIF in the cytoplasm of U937 cells. These results readily indicate that although it was selected by rMIF, the $\mathrm{HuScFv}$ molecules interacted with its target MIF antigen in the native form.

The tautomerase-active site is essential for the proinflammatory activity of MIF; thus, attempts have been made to develop tautomerase-neutralizing inhibitors $(21,40)$. A number of these inhibitors have also been proven beneficial for therapeutic efficacy in MIF-associated diseases (24,40-42). In the present study, inhibition of dopachrome tautomerization was performed to assess inhibition of MIF tautomerase activity mediated by $\mathrm{HuScFv}$. Subsequent to pre-incubation with MIF-specific HuScFv, the tautomerization activity of rMIF and native MIF, as detected by substrate decolorization, was reduced in a dose-dependent manner, when compared to that of the non-HuScFv control reaction, indicating its specific inhibition to MIF enzymatic activity. The finding that $\mathrm{HuScFv}$ 
inhibited the tautomerase activity of rMIF to a greater extent than that of native MIF may indicate the presence of other proteins containing the tautomerase activities in U937 cell lysate (2). In vitro neutralization of MIF-mediated enzymatic activity suggested the possibility of using $\mathrm{HuScFv}$ to reduce MIF-induced pro-inflammatory reactions, similar to the inhibitors or specific monoclonal antibodies as demonstrated in previous studies $(21,43)$. Experiments on in vivo neutralization tests are required to evaluate anti-inflammatory activities of MIF-specific HuScFv (30).

The HuScFv and MIF interaction was elaborated by the target epitope searching and molecular docking approaches. The 12-mer consensus mimotope sequence of MIF-specific $\mathrm{HuScFv}$ is located at the amino acid residues 22-33 of human MIF residing within the tautomerase active site. Molecular docking also revealed that the interaction between $\mathrm{HuScFv}$ and MIF spontaneously occurred with low-binding energy at residues Lys32 and Ile64 of the MIF tautomerase active site. This finding strongly supports the previous description of tautomerase catalytic residues including Pro1, Lys32, Ile64, Tyr95 and Asn97 (40). The phenomenon of the $\mathrm{HuScFv-mediated} \mathrm{inhibition} \mathrm{of} \mathrm{MIF} \mathrm{tautomerase} \mathrm{activity} \mathrm{is}$ similar to that exerted by the ISO1 chemical inhibitor (40).

In conclusion, $\mathrm{HuScFv}$ selected by using recombinant human MIF readily recognized native MIF present in human monoblastic leukemia (U937) cells. The antibody also neutralized tautomerase activity mediated by both rMIF and native MIF as it interacted with catalytic residues residing in the tautomerase active site of the MIF molecule. The inhibitory effect was elaborated by mimotope searching and the molecular docking approaches. MIF-specific HuScFv, with its fully-human format and small size, should be investigated in subsequent experiments to demonstrate its anti-inflammatory activity and its ability to develop into a therapeutic molecule for the treatment of human inflammatory diseases.

\section{Acknowledgements}

MT and OP are TRF young research scholars (supporting grant nos. TRG5680057 and TRG5480006, respectively). WC is supported by the research grant under the National Research University (NRU) Project, Office of Higher Education Commission (OHEC). PY is a TRF-Senior Research Scholar and also supported by Chalermphrakiat Grant, Faculty of Medicine Siriraj Hospital, Mahidol University. The authors would like to thank the National Nanotechnology Center (NANOTEC), the National Science and Technology Development Agency (NSTDA) for providing the Discovery Studio Package 2.5. The human antibody phage display library used in this study is the property of the National Research Council of Thailand (NRCT).

\section{References}

1. David JR: Delayed hypersensitivity in vitro: its mediation by cell-free substances formed by lymphoid cell-antigen interaction. Proc Natl Acad Sci USA 56: 72-77, 1966.

2. Merk M,Zierow S, Leng L, et al: The $D$-dopachrome tautomerase (DDT) gene product is a cytokine and functional homolog of macrophage migration inhibitory factor (MIF). Proc Natl Acad Sci USA 108: E577-E585, 2011.
3. Calandra $\mathrm{T}$ and Roger T: Macrophage migration inhibitory factor: a regulator of innate immunity. Nat Rev Immunol 3: 791-800, 2003.

4. Bernhagen J, Mitchell RA, Calandra T, Voelter W, Cerami A and Bucala R: Purification, bioactivity, and secondary structure analysis of mouse and human macrophage migration inhibitory factor (MIF). Biochemistry 33: 14144-14155, 1994.

5. Mitchell RA, Metz CN, Peng T and Bucala R: Sustained mitogenactivated protein kinase (MAPK) and cytoplasmic phospholipase A2 activation by macrophage migration inhibitory factor (MIF). Regulatory role in cell proliferation and glucocorticoid action. J Biol Chem 274: 18100-18106, 1999.

6. Mitchell RA, Liao H, Chesney J, Fingerle-Rowson G, Baugh J, David $\mathbf{J}$ and Bucala R: Macrophage migration inhibitory factor (MIF) sustains macrophage proinflammatory function by inhibiting p53: regulatory role in the innate immune response. Proc Natl Acad Sci USA 99: 345-350, 2002.

7. Sugimoto H, Taniguchi M, Nakagawa A, Tanaka I, Suzuki M and Nishihira J: Crystal structure of human $D$-dopachrome tautomerase, a homologue of macrophage migration inhibitory factor, at 1.54 A resolution. Biochemistry 38: 3268-3279, 1999.

8. Rosengren E, Aman P, Thelin S, et al: The macrophage migration inhibitory factor MIF is a phenylpyruvate tautomerase. FEBS Lett 417: 85-88, 1997.

9. Kleemann R, Kapurniotu A, Frank RW, et al: Disulfide analysis reveals a role for macrophage migration inhibitory factor (MIF) as thiol-protein oxidoreductase. J Mol Biol 280: 85-102, 1998.

10. Bozza FA, Gomes RN, Japiassu AM, Soares M, CastroFaria-Neto HC, Bozza PT and Bozza MT: Macrophage migration inhibitory factor levels correlate with fatal outcome in sepsis. Shock 22: 309-313, 2004.

11. Chen LC, Lei HY, Liu CC, et al: Correlation of serum levels of macrophage migration inhibitory factor with disease severity and clinical outcome in dengue patients. Am J Trop Med Hyg 74: 142-147, 2006.

12. Assunção-Miranda I, Amaral FA, Bozza FA, et al: Contribution of macrophage migration inhibitory factor to the pathogenesis of dengue virus infection. FASEB J 24: 218-228, 2010.

13. Hou XQ, Gao YW, Yang ST, Wang CY, Ma ZY and Xia XZ: Role of macrophage migration inhibitory factor in influenza $\mathrm{H} 5 \mathrm{~N} 1$ virus pneumonia. Acta Virol 53: 225-231, 2009.

14. Kim HR, Park MK, Cho ML, et al: Macrophage migration inhibitory factor upregulates angiogenic factors and correlates with clinical measures in rheumatoid arthritis. J Rheumatol 34: 927-936, 2007.

15. Foote A, Briganti EM, Kipen Y, Santos L, Leech M and Morand EF: Macrophage migration inhibitory factor in systemic lupus erythematosus. J Rheumatol 31: 268-273, 2004.

16. Lan HY, Yang N, Nikolic-Paterson DJ, et al: Expression of macrophage migration inhibitory factor in human glomerulonephritis. Kidney Int 57: 499-509, 2000.

17. Niino M, Ogata A, Kikuchi S, Tashiro $K$ and Nishihira J: Macrophage migration inhibitory factor in the cerebrospinal fluid of patients with conventional and optic-spinal forms of multiple sclerosis and neuro-Behçet's disease. J Neurol Sci 179: 127-131, 2000.

18. Burger-Kentischer A, Goebel H, Seiler R, et al: Expression of macrophage migration inhibitory factor in different stages of human atherosclerosis. Circulation 105: 1561-1566, 2002.

19. Herder C, Kolb H, Koenig W, et al: Association of systemic concentrations of macrophage migration inhibitory factor with impaired glucose tolerance and type 2 diabetes: results from the Cooperative Health Research in the Region of Augsburg, Survey 4 (KORA S4). Diabetes Care 29: 368-371, 2006.

20. Bucala R and Donnelly SC: Macrophage migration inhibitory factor: a probable link between inflammation and cancer. Immunity 26: 281-285, 2007.

21. Al-Abed Y, Dabideen D, Aljabari B, et al: ISO-1 binding to the tautomerase active site of MIF inhibits its pro-inflammatory activity and increases survival in severe sepsis. J Biol Chem 280: 36541-36544, 2005.

22. Cvetkovic I, Al-Abed Y, Miljkovic D, et al: Critical role of macrophage migration inhibitory factor activity in experimental autoimmune diabetes. Endocrinology 146: 2942-2951, 2005.

23. Nicoletti F, Créange A, Orlikowski D, et al: Macrophage migration inhibitory factor (MIF) seems crucially involved in Guillain-Barré syndrome and experimental allergic neuritis. J Neuroimmunol 168: 168-174, 2005. 
24. Alam A,Haldar S, Thulasiram HV, et al: Novel anti-inflammatory activity of epoxyazadiradione against macrophage migration inhibitory factor: inhibition of tautomerase and proinflammatory activities of macrophage migration inhibitory factor. J Biol Chem 287: 24844-24861, 2012.

25. Calandra T, Echtenacher B, Roy DL, et al: Protection from septic shock by neutralization of macrophage migration inhibitory factor. Nat Med 6: 164-170, 2000.

26. Bernhagen J, Bacher M, Calandra T, Metz CN, Doty SB, Donnelly $\mathrm{T}$ and Bucala R: An essential role for macrophage migration inhibitory factor in the tuberculin delayed-type hypersensitivity reaction. J Exp Med 183: 277-282, 1996.

27. Mikulowska A, Metz CN, Bucala R and Holmdahl R: Macrophage migration inhibitory factor is involved in the pathogenesis of collagen type II-induced arthritis in mice. J Immunol 158: 5514-5517, 1997.

28. Cvetkovic I and Stosic-Grujicic S: Neutralization of macrophage migration inhibitory factor-novel approach for the treatment of immunoinflammatory disorders. Int Immunopharmacol 6: $1527-1534,2006$

29. Greven D, Leng L and Bucala R: Autoimmune diseases: MIF as a therapeutic target. Expert Opin Ther Targets 14: 253-264, 2010

30. Kerschbaumer RJ, Rieger M, Völkel D, et al: Neutralization of macrophage migration inhibitory factor (MIF) by fully human antibodies correlates with their specificity for the $\beta$-sheet structure of MIF. J Biol Chem 287: 7446-7455, 2012.

31. Kulkeaw K, Sakolvaree Y, Srimanote P, et al: Human monoclonal ScFv neutralize lethal Thai cobra, Naja kaouthia, neurotoxin. J Proteomics 72: 270-282, 2009.

32. Poungpair O, Pootong A, Maneewatch S, et al: A human single chain transbody specific to matrix protein (M1) interferes with the replication of influenza A virus. Bioconjug Chem 21: 1134-1141, 2010.

33. Dios A, Mitchell RA, Aljabari B, et al: Inhibition of MIF bioactivity by rational design of pharmacological inhibitors of MIF tautomerase activity. J Med Chem 45: 2410-2416, 2002.
34. Thanongsaksrikul J, Srimanote $\mathrm{P}$, Maneewatch S, et al: $\mathrm{A} \mathrm{V}^{\mathrm{H}} \mathrm{H}$ that neutralizes the zinc metalloproteinase activity of botulinum neurotoxin type A. J Biol Chem 285: 9657-9666, 2010.

35. Laskowski RA, MacArthur MW, Moss DS and Thornton JM PROCHECK: a program to check the stereochemical quality of protein structures. J Appl Cryst 26: 283-291, 1993.

36. Grieb G, Merk M, Bernhagen J and Bucala R: Macrophage migration inhibitory factor (MIF): a promising biomarker. Drug News Perspect 23: 257-264, 2010.

37. Dagia NM, Kamath DV, Bhatt P, et al: A fluorinated analog of ISO-1 blocks the recognition and biological function of MIF and is orally efficacious in a murine model of colitis. Eur J Pharmacol 607: 201-212, 2009.

38. Thathaisong U, Maneewatch S, Kulkeaw K, et al: Human monoclonal single chain antibodies ( $\mathrm{HuScFv}$ ) that bind to the polymerase proteins of influenza A virus. Asian Pac J Allergy Immunol 26: 23-35, 2008

39. Maneewatch S, Thanongsaksrikul J, Songserm T, et al: Human single-chain antibodies that neutralize homologous and heterologous stains and clades of influenza A virus subtype H5N1. Antivir Ther 14: 221-230, 2009.

40. Lubetsky JB, Dios A, Han J, et al: The tautomerase active site of macrophage migration inhibitory factor is a potential target for discovery of novel anti-inflammatory agents. J Biol Chem 277: 24976-24982, 2002.

41. Al-Abed Y and Van Patten S: MIF as a disease target: ISO-1 as a proof-of-concept therapeutic. Future Med Chem 3: 45-63, 2011.

42. Kithcart AP, Cox GM, Sielecki T, et al: A small-molecule inhibitor of macrophage migration inhibitory factor for the treatment of inflammatory disease. FASEB J 24: 4459-4466, 2010.

43. Zhang Y, Zeng X, Chen S, et al: Characterization, epitope identification and mechanisms of the anti-septic capacity of monoclonal antibodies against macrophage migration inhibitory factor. Int Immunopharmacol 11: 1333-1340, 2011. 